\title{
ADAMTS13 deficiency and immunological abnormalities in patients with systemic sclerosis
}

\author{
Zofia Gerlicz-Kowalczuk¹, Jolanta D. Torzecka², Elżbieta Dziankowska-Zaborszczyk³, Alicja Ograczyk ${ }^{1}$, \\ Anna M. Zalewska-Janowska ${ }^{1}$, Anna Woźnicka², Bożena Dziankowska-Bartkowiak²
}

${ }^{1}$ Department of Psychodermatology, Medical University of Lodz, Lodz, Poland

${ }^{2}$ Department of Dermatology and Venereology, Medical University of Lodz, Lodz, Poland

${ }^{3}$ Department of Preventive Medicine, Medical University of Lodz, Lodz, Poland

Adv Dermatol Allergol 2018; XXXV (2): 182-184

DOI: https://doi.org/10.5114/ada.2018.75240

\begin{abstract}
Introduction: Systemic sclerosis (SSc) is a chronic autoimmune connective tissue disorder characterized by immunological deviations and generalized microvascular damage.

Aim: To determine the serum level of the von Willebrand factor cleaving protease (ADAMTS13) in 39 SSc patients and healthy controls.

Material and methods: ADAMTS13 serum level was determined in 39 SSc patients and 11 healthy controls. Complete history of the patients was recorded and thorough clinical, rheumatological, and dermatological examinations were performed.

Results: The serum levels of ADAMTS13 were significantly lower in SSc than in normal controls $(455.47 \pm 128$ vs. $702.01 \pm 142 \mathrm{ng} / \mathrm{ml}, p<0.00001$ ). However significant correlations among serum ADAMTS 13 levels and organ changes were not found in SSc patients.

Conclusions: We demonstrate a decreased serum level of ADAMTS13 in SSc patients, which may contribute to the vessel microangiopathy observed in systemic sclerosis.
\end{abstract}

Key words: systemic sclerosis, ADAMTST13.

\section{Introduction}

The pathogenesis of systemic sclerosis is still unknown, although immunological deviations, generalized microcirculation disorders and progressive tissue fibrosis may have an important role in initiating and perpetuating the disease [1]. At present the role of initial morphological and functional markers of systemic sclerosis in microvascular structural damage have been put forward. ADAMTS13 (a disintegrin and metalloproteinase with thrombospondin type 1 motives) is a zinc-containing metalloprotease that is involved in cleavage of von Willebrand factor and is synthesized by endothelial cells and megakaryocytes [2]. Deficiency of von Willebrand factor cleaving protease (ADAMTS13) may contribute to endothelial injury followed by vessel damage and subsequent fibrosis [3, 4]. However, in the pathogenesis of systemic sclerosis (SSc) there is considerable mechanistic overlap, and the vessel injury and fibrosis probably have multifactorial causation but ADAMTS13 may play an important role as a co-factor.
Aim

Thus, the aim of the study was to evaluate the concentration of the ADAMTS13 and attempt to evaluate their role in the pathogenesis of SSc. An attempt was also made to assess the relationship between the concentration of the selected parameters and the diagnosed organ changes as well as vascular disorders observed in capillaroscopy.

\section{Material and methods}

Blood samples were obtained from 39 SSc patients (37 females and 2 male; 29 limited systemic sclerosis (IcSSc), 10 diffuse systemic sclerosis (dcSSc) according to the criteria of LeRoy [5] and 11 healthy individuals, all participants have signed informed consent approved by the Ethical Committee. All SSc patients fulfilled the criteria of the American College of Rheumatology (1980) [6]. The control group consisted of 11 randomly selected healthy subjects with no systemic diseases or on medica-

Address for correspondence: Zofia Gerlicz-Kowalczuk, Department of Psychodermatology, Medical University of Lodz, 251 Pomorska St build. C5, 92-213 Lodz, Poland, phone: +48 606168 467, e-mail: zosia_gerlicz@yahoo.com Received: 26.09.2016, accepted: 24.01.2017. 
tion. All samples were taken between 7:00 and 9:00 a.m. The samples were centrifuged and the obtained sera were stored in aliquots at $-20^{\circ} \mathrm{C}$ until analyses. Clinical, laboratory and treatment data were collected at the time when the blood samples were drawn. Drugs, including corticosteroids and immunosuppressants, were authorized but stopped $24 \mathrm{~h}$ before blood collection. All patients with SSc were taking vasodilators and some of them corticosteroids (15 patients: 9 with lcSSc, 6 with dcSSc) or/and methotrexate (4 patients: 3 with lcSSc, 1 with dcSSc) or/and cyclophosphamide ( 5 patients: 3 with IcSSc, 2 with dcSSc). The patient characteristics are presented in Table 1.

\section{Clinical assessment}

An extensive clinical profile was established for each SSc patient [7]. The history and complete physical examination were obtained from each patient. The patients were evaluated for the cardiac (diagnosed by Holter, ECG, echocardiography and cardiological consultation), pulmonary (chest RTG, high-resolution computed tomography (HRCT) scan of thorax and pulmonary consultation), esophageal (esophageal scintigraphy), renal and hematological (blood test, bone marrow biopsy if needed) involvement. Cardiovascular changes included abnormal cardiac rhythm or documented fibrosis of myocardium. Pulmonary involvement was defined as the presence of ground glass or honeycombing appearances that suggest lung fibrosis. Esophageal changes were diagnosed on the basis of impaired transit in scintigraphy. Renal involvement was found if serum creatinine was above $100 \mu \mathrm{mol} / \mathrm{l}$, significant proteinuria - above $500 \mathrm{mg}$ for $24 \mathrm{~h}$ (after excluding other causes) or documented renal crisis in the past. Hematological involvement was diagnosed in case of leukopenia or anemia, after excluding other causes. Medical history of the disease (onset of Raynaud's phenomenon and skin sclerosis), ulcerations in finger tip pulp and the medications in the past and at present were also considered. Duration of the disease has been established since the skin sclerosis.

Levels of ADAMTS13 were determined by quantitative colorimetric sandwich ELISA kits (R\&D Systems, Minneapolis, MN, USA) strictly according to the manufacturer's instructions.

\section{Statistical analysis}

Statistical significance was analyzed by non-parametric Mann-Whitney test, Kruskal-Wallis test, Student's t-test and Spearman's rank correlation test. P-values less than 0.05 were considered significant.

\section{Results}

Mean ADAMTS13 serum levels were decreased in SSc patients compared to healthy controls (455.47 \pm 128 vs. $702.01 \pm 142 \mathrm{ng} / \mathrm{ml}, p<0.00001)$. However serum lev- els of ADAMTS13 were not different between ISSC and dSSc. Significant correlations between serum levels of ADAMTS13 and organ changes were not found in SSC patients, as well as serum levels of ADAMTS13 and duration of the Raynaud's phenomenon or disease, skin involvement or taking immunosuppressive drugs. Interestingly, the Kruskal-Wallis test in SSc subsets showed that ADAMTS13 serum levels were associated with elevated antinuclear antibodies (ANA) (Table 2).

\section{Discussion}

The data obtained in our study showed decreased plasma levels of ADAMTS13 in SSc patients. Molecular mechanism of hemostatic balance and thrombotic microangiopathies is still poorly understood. However, pathogenesis of SSc may be initiated in the vasculature, hence reduced activity of ADAMTS13 may additionally promote a prothrombotic function of von Willebrand factor (VWF) and can be an important factor triggering progression of blood vessel damage and consequent fibrosis [8]. Studies suggested also that endothelial cells secrete ADAMTS13, although its impact on vasculature has not been established $[9,10]$.

Table 1. Clinical characteristics of 39 patients with systemic sclerosis

\begin{tabular}{|c|c|c|c|}
\hline \multirow[t]{2}{*}{ Parameter } & \multicolumn{3}{|c|}{ Number/percentage or mean \pm SD } \\
\hline & SSc & IcSS & dcSS \\
\hline Age at study [years] & $55 \pm 10.9$ & $56 \pm 12.01$ & $53 \pm 7.42$ \\
\hline Age of onset [years] & $42 \pm 9.79$ & $41 \pm 9.94$ & $45 \pm 8.88$ \\
\hline $\begin{array}{l}\text { Duration of the skin } \\
\text { sclerosis [years] }\end{array}$ & $8 \pm 6.18$ & $9 \pm 6.50$ & $6 \pm 4.72$ \\
\hline Female/male ratio & $\begin{array}{c}37 / 2 \\
(5 \% / 95 \%)\end{array}$ & $\begin{array}{c}28 / 1 \\
(96 \% / 4 \%)\end{array}$ & $\begin{array}{c}9 / 1 \\
(90 \% / 10 \%)\end{array}$ \\
\hline Limited/diffuse SSc & $\begin{array}{c}29 / 10 \\
(74 \% / 26 \%)\end{array}$ & & \\
\hline ANA: & $100 \%$ & & \\
\hline Anti-topoisomerase I & $55 \%$ & $47 \%$ & $80 \%$ \\
\hline Anti-centromere & $18 \%$ & $24 \%$ & $0 \%$ \\
\hline U1RNP & $15 \%$ & $21 \%$ & $0 \%$ \\
\hline Ro-52 & $21 \%$ & $21 \%$ & $20 \%$ \\
\hline Duration of the RP [years] & $13 \pm 7.19$ & $15 \pm 7.06$ & $7 \pm 4.70$ \\
\hline Organ involvement & $85 \%$ & $83 \%$ & $90 \%$ \\
\hline Cardiac involvement & $79 \%$ & $83 \%$ & $70 \%$ \\
\hline Pulmonary involvement & $33 \%$ & $31 \%$ & $40 \%$ \\
\hline Esophagus involvement & $61 \%$ & $59 \%$ & $70 \%$ \\
\hline Renal involvement & $13 \%$ & $7 \%$ & $30 \%$ \\
\hline Pitting scars & $69 \%$ & $69 \%$ & $70 \%$ \\
\hline
\end{tabular}


Z. Gerlicz-Kowalczuk, J.D. Torzecka, E. Dziankowska-Zaborszczyk, A. Ograczyk, A.M. Zalewska-Janowska, A. Woźnicka, B. Dziankowska-Bartkowiak

Table 2. Serum levels of ADAMTS13

\begin{tabular}{|c|c|c|c|c|c|}
\hline \multirow[t]{2}{*}{ Parameter } & \multirow[t]{2}{*}{ Statistical data } & \multirow{2}{*}{$\begin{array}{c}\text { SSc } \\
(n=39)\end{array}$} & \multicolumn{2}{|c|}{ Investigated groups } & \multirow{2}{*}{$\begin{array}{l}\text { Control group } \\
\quad(n=14)\end{array}$} \\
\hline & & & $\operatorname{IcSSc}(n=29)$ & $\operatorname{dcSSc}(n=10)$ & \\
\hline \multirow{3}{*}{$\begin{array}{l}\text { ADAMTS13 } \\
{[\mathrm{ng} / \mathrm{ml}]}\end{array}$} & $\bar{x}$ & 466.47 & 468.45 & 460.74 & 702.01 \\
\hline & Range (min.-max.) & $211-768$ & $211-768$ & $276-672$ & $478-1028$ \\
\hline & SD & 128.03 & 136.56 & 105.57 & 142.36 \\
\hline \multirow[t]{4}{*}{ ANA } & & $1 / 640(n=7)$ & $1 / 1280(n=12)$ & $1 / 2560(n=20)$ & \\
\hline & $\bar{x}$ & 538.88 & 439.05 & 468.12 & \\
\hline & Range (min.-max.) & $394-768$ & $211-672$ & 125.46 & \\
\hline & SD & 146.41 & $276-764$ & 131.08 & \\
\hline \multirow[t]{4}{*}{ Ro-52 } & & $\begin{array}{l}\text { Present } \\
(n=9)\end{array}$ & $\begin{array}{l}\text { Absent } \\
(n=30)\end{array}$ & & \\
\hline & $\bar{x}$ & 538.87 & 448.77 & & \\
\hline & Range (min.-max.) & $433-695$ & $211-768$ & & \\
\hline & SD & 101.95 & 133.21 & & \\
\hline
\end{tabular}

Moreover Mannucci et al. has not found antiADAMTS13 in SSc patients or in the investigated systemic lupus erythematosus (SLE) groups [11], suggesting that some other changes may be decreasing the level of ADAMTS13 in autoimmunological connective diseases, as endothelium damage. Scheja et al. study has suggested that von Willebrand factor (VWF) propeptide may be useful in the assessment of disease activity in SSc [12] However in our study, relationships between ADAMTS13 and organ involvement, as well as prominent markers of endothelial injury, as Raynaud's phenomenon or finger tips ulcers were not observed.

This study seems to confirm the correlation between immunological abnormalities and decreased ADAMTS13 serum levels suggesting that reduced activity of ADAMTS13 is contributing to enhance the prothrombotic function of VWF and may play a role in microangiopathy and secondary fibrosis.

\section{Conclusions}

In this study, we demonstrated that ADAMTS13 serum levels are decreased in SSC and associated with the elevated antinuclear antibody level in SSc patients and presence of Ro-52 antibodies. Taken together our data further underscore the role of dysregulated hemostasis in SSC.

\section{Conflict of interest}

The authors declare no conflict of interest.

\section{References}

1. Abraham DJ, Krieg T, Distler J, Distler O. Overview of pathogenesis of systemic sclerosis. Rheumatology (Oxford) 2009; 48: iii3-7.
2. Franchini M, Montagnana M, Targher G, Lippi G. Reduced von Willebrand factor-cleaving protease levels in secondary thrombotic microangiopathies and other diseases. Semin Thromb Hemost 2007; 33: 787-97.

3. Lip GY, Blann A. von Willebrand factor: a marker of endothelial dysfunction in vascular disorders? Cardiovasc Res 1997; 34: 255-65.

4. Ruiz-Torres MP, Casiraghi F, Galbusera M, et al. Complement activation: the missing link between ADAMTS-13 deficiency and microvascular thrombosis of thrombotic microangiopathies. Thromb Haemost 2005; 93: 443-52.

5. LeRoy E, Black C, Fleischmajer R, et al. Scleroderma (systemic sclerosis): classification, subsets and pathogenesis. J Rheumatol 1988; 15: 202-5.

6. Masy AT, Rodnan GP, Medsger TA, et al. Diagnostic and Therapeutic Criteria Committee (1980). Preliminary criteria for the classification of systemic sclerosis (scleroderma). Arthritis Rheum 1980; 23: 581-90.

7. Gerlicz Z, Dziankowska-Bartkowiak B, Dziankowska-Zaborszczyk E, Sysa-Jedrzejowska A. Disturbed balance between serum levels of receptor tyrosine kinases Tie-1, Tie-2 and angiopoietins in systemic sclerosis. Dermatology 2014; 228: 233-9.

8. Qin F, Impeduglia T, Schaffer P, Dardik H. Overexpression of von Willebrand factor is an independent risk factor for pathogenesis of intimal hyperplasia: preliminary studies. J Vasc Surg 2003; 37: 433-9.

9. Lee M, Rodansky ES, Smith JK, Rodgers GM. ADAMTS13 promotes angiogenesis and modulates VEGF-induced angiogenesis. Microvasc Res 2012; 84: 109-15.

10. Kling SJ, Judd CA, Warner KB, Rodgers GM. Regulation of ADAMTS13 expression in proliferating human umbilical vein endothelial cells. Pathophysiol Haemost Thromb 2008; 36: 233-40.

11. Mannucci PM, Vanoli M, Forza I, et al. Von Willebrand factor cleaving protease (ADAMTS-13) in 123 patients with connective tissue diseases (systemic lupus erythematosus and systemic sclerosis). Haematologica 2003; 88: 914-8.

12. Scheja A, Akesson A, Geborek, et al. Von Willebrand factor propeptide as a marker of disease activity in systemic sclerosis (scleroderma). Arthritis Res 2001; 3: 178-82. 